\title{
ROBOTIC COMPUTER-ASSISTED TELEMANIPULATION ENHANCES CORONARY ARTERY BYPASS
}

Hani Shennib, MD

Amr Bastawisy, MD

Joan McLoughlin, RN*

Frederic Moll, MD*
Objectives: Totally endoscopic coronary artery bypass grafting depends greatly on perfecting the anastomosis. We tested a new computer-assisted telemanipulation robot (Intuitive Surgical Inc, Mountain View, Calif) in performing endoscopic coronary bypass. Methods: On-bench anastomoses of the porcine arterial graft to the left anterior descending coronary artery were performed with both direct visualization and conventional surgical instruments (group I), endoscopic 3-dimensional visualization and current endoscopic surgical instruments (group II), direct visualization and endoscopic instruments (group III), 3-dimensional endoscopic visualization and conventional surgical instruments (group IV), and telemanipulation robotic with 3-dimensional endoscopic visualization (group V). Anastomoses ( $n=6$ in each group) were assessed for time (minutes), quality ( $\operatorname{good}=3$, fair $=2$, poor $=1$ ), technical difficulty (easy-difficult: $1-4)$, and patency $(100 \%=1,>50 \%=2$, $<50 \%=3)$. Results: Anastomotic time was significantly longer in groups II and III than in groups IV and V $(P \leq .02)$. Patency was comparable in all groups. Conclusion: Telemanipulation technology may enhance the performance of totally endoscopic coronary artery anastomosis. The facility and time of an Intuitive telemanipulation anastomosis is comparable with that of a conventional anastomosis created under direct vision. (J Thorac Cardiovasc Surg 1999;117:310-3)
$\mathrm{M}$ inimally invasive coronary artery bypass graft surgery has been used increasingly over the past 3 years. ${ }^{1}$ In general, it is agreed that a procedure that eliminates cardiopulmonary bypass and/or decreases tissue dissection and incision is minimally invasive.

The challenge of totally endoscopic coronary artery bypass surgery is great. Adequate visualization of the vascular graft and the recipient arteries from a variety of angles to permit precise anastomosis is a limiting factor. The instruments currently used for endoscopic suturing are crude and difficult to manipulate. The use of straight instruments positioned on a fulcrum forces surgeons to perform reverse movements, and the distance between

From the Department of Cardiothoracic Surgery, McGill University, Montreal, Quebec, Canada.

Received for publication May 1, 1998; revisions requested May 22, 1998; revisions received July 3, 1998; accepted for publication Oct 1, 1998.

Address for reprints: Hani Shennib, MD, The Montreal General Hospital, 1650 Cedar Ave, Suite L9-121, Montreal, Quebec H3G 1A4, Canada.

*Frederic Moll is Medical Director and Joan McLoughlin is an employee of Intuitive Surgical Inc, Mountain View, California.

Copyright (C) 1999 by Mosby, Inc.

0022-5223/99 $\$ 8.00+0 \quad \mathbf{1 2 / 1 / 9 5 2 9 7}$ the working point and fulcrum decreases dexterity and increases the risk of tremors. Magnification of the field augments tremors and renders the whole technique awkward and uncomfortable. Finally, the anatomic location of the coronary arteries and the stiffness of the chest wall limit access through small incisions.

Recently, telemanipulation technology has been implemented in abdominal surgery to increase surgical dexterity and precision. ${ }^{2}$ Although a variety of telemanipulation and robotically assisted technologies exist, 3,4 we have elected to explore that potential for enhancing coronary artery bypass surgery using an advanced technology developed by Intuitive Surgical, Inc, Mountain View, California. This technology provides 3-dimensional imaging for better perception of depth and optical resolution. By providing 7 scales of motion, it permits the surgeon to manipulate instruments inside the chest and exercise digital and wrist movements via instruments inserted through minute $10-\mathrm{mm}$ incisions. The surgeon manipulates the instruments by sitting at a console at a distance from the operating room table. In this study, we explore the feasibility of performing totally endoscopic minimally invasive coronary artery bypass graft surgery by using such telemanipulation technology. 


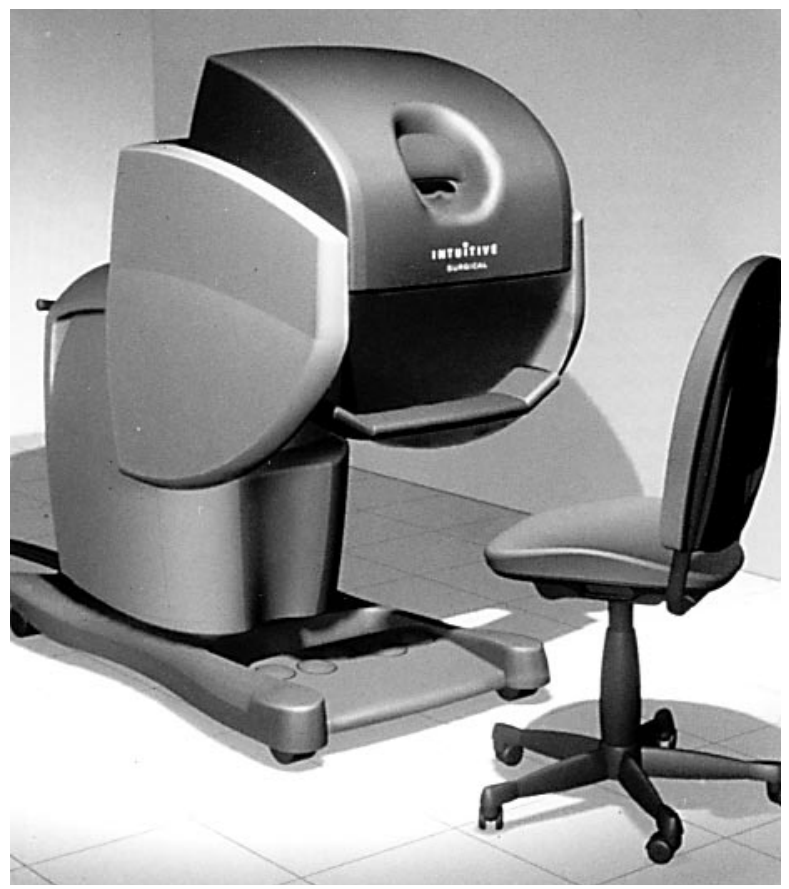

Fig 1. The surgeon's console with elbow support and 3dimensional viewer.

\section{Material and methods}

Five groups of porcine hearts ( $n=6$ in each) were prepared by dissecting the circumflex artery, which was used as a free graft to the left anterior descending artery in an end-to-side anastomosis with 7-0 Prolene continuous sutures (Ethicon, Inc, Somerville, NJ). The anastomoses were done with the heart positioned in a closed cardboard box with access through 12-mm endoports (Ethicon Endo-Surgery, Inc, Cincinnati, Ohio). All anastomoses were performed by one fully trained cardiothoracic surgeon. In group I, anastomoses were performed with conventional coronary surgical instruments under ordinary 3-dimensional visualization by surgical loupes. In group II, the anastomoses were done with reusable endoscopic microvascular instruments (Pilling Weck Surgical, Fort Washington, $\mathrm{Pa}$ ) and indirect visualization with commercially available VISTA 3-dimensional head-mounted display (VISTA Cardiothoracic System, Westborough, Mass). In group III, the anastomoses were performed with direct visualization by ordinary surgical loupes and endoscopic instruments. In group IV, conventional instruments were used to perform the anastomoses with 3-dimensional head-mounted display. In group V, the anastomoses were performed with an advanced telemanipulation system (Intuitive Surgical). The telemanipulation system consists of 3 parts: (1) the surgeon's console (Fig 1); (2) the patient's side, which is set up with joint arms (Fig 2); (3) the slave instruments, which are $55 \mathrm{~cm}$ long and $8.4 \mathrm{~mm}$ in diameter and permit insertion through ports that are $10 \mathrm{~mm}$ in diameter. The oval distance tips mea-

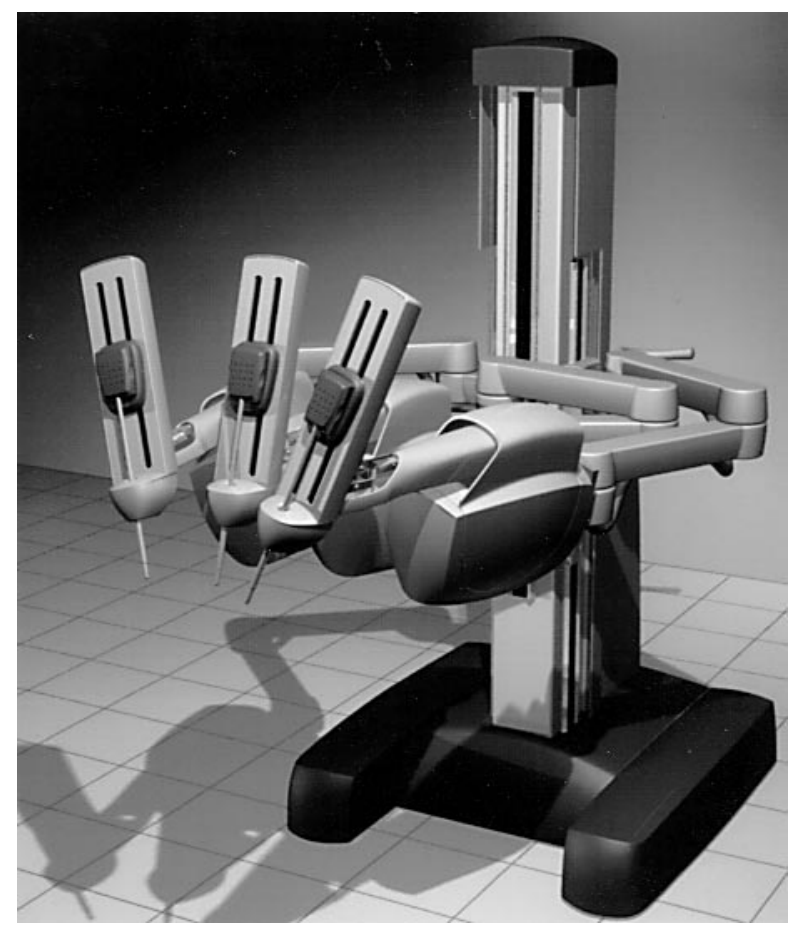

Fig 2. The robotic arms: Two manipulate the surgical instruments and the third controls the thoracoscope. The endoscopic instruments are then attached to the arms by the assistant.

sure $6.1 \times 6.4 \mathrm{~mm}$ in diameter and are approximately $7 \mathrm{~mm}$ in length (Fig 3). A 3-dimensional viewer is an integral part of the system. An illustration of the various components and its relations to the heart preparation is shown in Fig 4.

All anastomoses were evaluated for the following: (1) time (in minutes) required for completion of the anastomosis from the first suture bite until the seventh knot; (2) surgeon's satisfaction with quality of the anastomosis at completion (good = 3 , fair $=2$, poor $=1)$; (3) intraoperative events (eg, fracture of the sutures, breaking of needles, injury to the internal thoracic artery or coronary artery; (4) degree of difficulty of anastomosis (easy $=1$, somewhat easy $=2$, somewhat difficult $=3$, difficult = 4); (5) graft patency, assessed by direct gross inspection and sized metal probing. Patency was graded as follows: $100 \%=1,50 \%=2$, less than $50 \%=3$. Statistical analysis was performed between groups by means of the Student $t$ test. Results are reported as mean \pm standard deviation.

\section{Results}

Results are shown in Table I. Anastomotic time was $6.7 \pm 0.5$ minutes for group I, $22.4 \pm 3.0$ for group II, $21.1 \pm 2.1$ for group III, $10.5 \pm 1.6$ for group IV, and 8.9 \pm 1.4 minutes for group V. It was significantly shorter in group I than in the other groups $(P \leq .04)$ and significantly longer in groups II and III than in groups IV and 


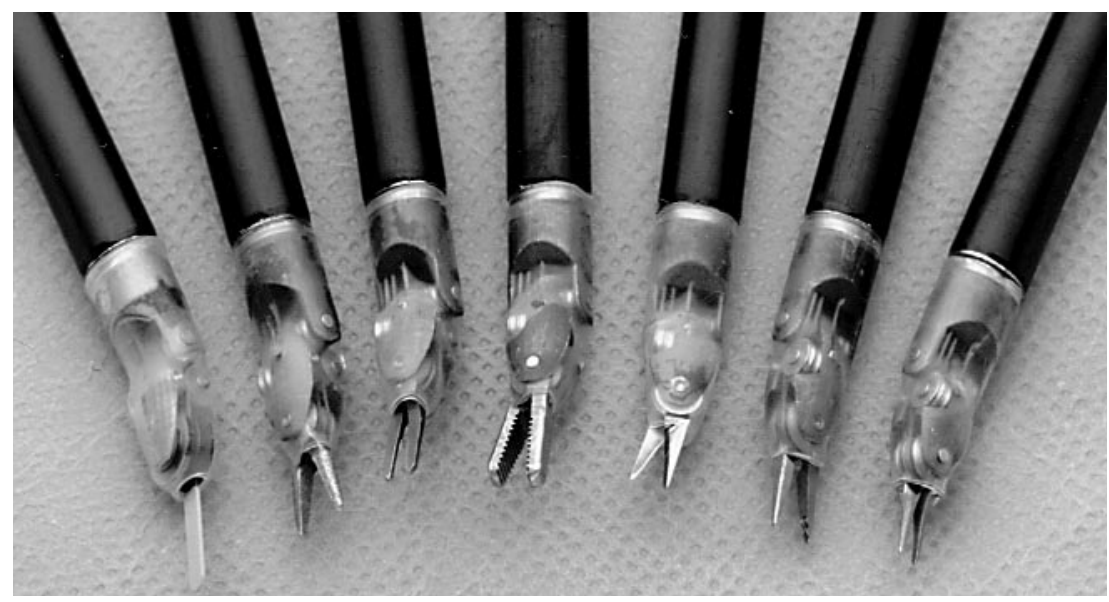

Fig 3. A variety of endoscopic instruments. Right to left, Mobile cautery tip, needle holder, clip applier, toothed grasper, 2 pairs of scissors, and a fine forceps.

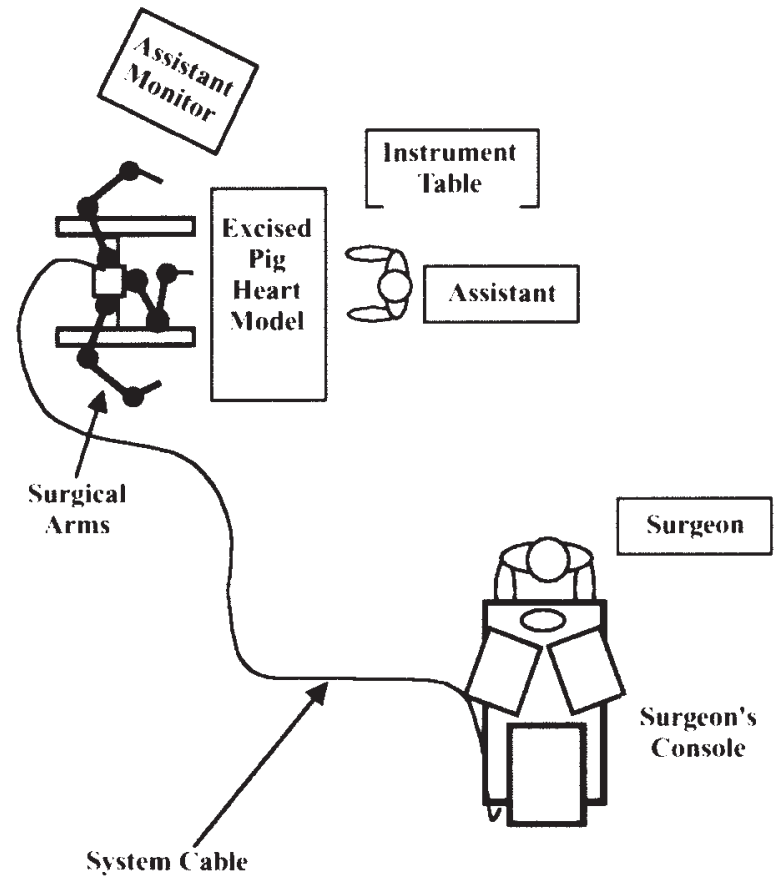

Fig 4. Diagram of the experimental setup showing the bench with the heart model, the robotic arms with the instrument tips, and the console where the surgeon sits to telemanipulate the instruments.

$\mathrm{V}(P \leq .02)$. Despite the observed statistically significant difference in time between group I (conventional instruments with direct visualization) and group V (telemanipulation technology under 3-dimensional visualization), with a $P$ value $\leq .004$, the times were comparable clinically (6.7 and 8.9 , respectively). The quality of the anastomoses was found to be inferior in group III compared with group I $(P \leq .04)$, in groups II and III compared with IV $(P \leq 0.02)$, and in group III compared with group $\mathrm{V}(P \leq 0.02)$. On the other hand, quality was comparable between groups I and V. The difficulty in performing the anastomosis as perceived by the surgeon was found to be significantly more in groups II and III than in groups I, IV, and V $(P \leq .02)$. Anastomoses were performed with comparable ease in groups I and V $(P \leq$ .21). Patency was similar in all groups.

\section{Discussion}

Endoscopic techniques are currently used in a wide range of general, gynecologic, thoracic, and orthopedic procedures. The advantages of minimal-access surgery have been clearly documented in the general surgical literature $^{5}$ : less morbidity, pain, cost, and length of hospital stay, in addition to faster recovery and return to work. Recently, there has been increasing interest in the use of minimally invasive coronary artery bypass grafting and valve surgery. Minimally invasive cardiac surgery is defined as a procedure done through smaller incisions with less tissue dissection and/or without use of cardiopulmonary bypass. Currently, less that $5 \%$ of cardiac procedures are done through minimally invasive approaches. These include single coronary artery bypass grafts performed through limited thoracotomy incisions and multivessel coronary artery bypass grafting done through sternotomy and other incisions with cardiopulmonary bypass. More recently, cardiac surgeons have applied endoscopic techniques for intraoperative diagnosis and for selected tasks during cardiac procedures. The improved visualization, when video 
Table I. Comparison of anastomotic timing, quality, technical difficulties, and patency between different techniques

\begin{tabular}{lllll}
\hline & Time $(\mathrm{min})$ & Quality & Difficulty & Anastomotic patency \\
\hline Group I (DV and CI) & $6.7 \pm 0.5$ & $2.8 \pm 0.5$ & $1.0 \pm 0.00 \dagger$ & $1.0 \pm 0.00$ \\
Group II (EV and EI) & $22.4 \pm 3.0^{*}$ & $1.8 \pm 1.0$ & $4.0 \pm 0.00$ & $1.5 \pm 0.8$ \\
Group III (DV and EI) & $21.1 \pm 2.1^{*}$ & $1.0 \pm 0.00^{*}$ & $4.0 \pm 0.00$ & $1.5 \pm 0.55$ \\
Group IV (EV and CI) & $10.5 \pm 1.6^{* \dagger}$ & $2.5 \pm 0.55 \dagger$ & $1.0 \pm 0.00 \dagger$ & $1.0 \pm 0.00$ \\
Group V (TR) & $8.87 \pm 1.44^{* \dagger}$ & $2.0 \pm 0.00 \ddagger$ & $1.3 \pm 0.5 \dagger$ & $1.0 \pm 0.00$ \\
\hline
\end{tabular}

Values are mean \pm SD. $D V$, Direct vision; $C I$, conventional instruments; $E V$, endoscopic visualization; $E I$, endoscopic instruments; $T R$, telemanipulation robotic technology. $* P \leq .04$ compared with group I.

$\dagger P \leq .02$ compared with groups II and III.

$\ddagger P \leq .02$ compared with group III

assistance is used, has the potential of better assessment of intracardiac abnormalities and more precise repair.

The performance of totally endoscopic coronary artery bypass grafting, however, remains elusive and technically demanding. Limited visualization, lack of suitable instrumentation, restricted anatomic access, and reluctance to accept new technology are among many factors that make totally endoscopic cardiac surgery unlikely today. Endoscopic cardiac surgery is particularly hindered by the current design of long endoscopic instruments and the need to manipulate the instruments with the surgeon's wrist outside the chest. This requires a high degree of skill and hand-eye coordination, particularly inasmuch as instruments are manipulated in the reverse direction of the intended action.

Telemanipulation technology with Intuitive devices is principled on a servo control system processing power technology. This is responsible for making a master handle and a slave to appear to be rigidly connected. This is accomplished by computing the locations of the two master handles within the surgeon's console, as well as corresponding slave tools within the patient: measuring the forces applied by the slave tool to the patient, the forces applied to the master handle by the surgeon, and determining the proper motor forces to be applied to make these two ends of the system appear to be intuitively connected. The servo control engine packs enough power to update the tools and master motions 2000 times every second. This amounts to 320 MSLOPS (million calculations per second). By comparison, a $50 \times 50$ cell spread sheet can contain 2500 calculations. If the servo control engine were applied to this desktop processing task, it could evaluate the spread sheet more than 100,000 times in a single second. In the future, the surgeon who sits on the console may be able to perform surgical tasks within the thoracic cavity under 3-dimensional vision and execute those tasks by using short instruments, with the wrist inside the patient, and a high degree of precision. The telemanipulation technology provides a wrist-like device with 7 degrees of freedom at the end of each instrument. The surgeon's visual emersion into the operating field with the 3-dimensional visualization system provides better hand-eye coordination and lineof-sight imaging and provides accessory feedback and motion scaling. Tremors are subsequently eliminated.

In this feasibility study, we noted a clear advantage of using Intuitive telemanipulation technology in performing on-bench coronary artery anastomosis. The timing, quality, and ease of the computer-assisted robotic anastomoses were superior to those done under direct vision with conventional instruments. We conclude that telemanipulation technology may be beneficial in performing totally endoscopic coronary artery bypass surgery.

\section{REFERENCES}

1. Shennib H, Mack M, Lee A. A survey on minimally invasive coronary artery bypass grafting. Ann Thorac Surg 1997;64:110-5.

2. Voges U. Laparoscopic techniques: Which developments are possible? Urologe A 1996;35:208-14.

3. Garcia-Ruiz A, Smerdira NG, Loop FD, Hahn JF, Miller MS, Steiner CP, et al. Robotic surgical instruments for dexterity enhancement in thoracoscopic coronary artery bypass graft. J Laparoendosc Adv Surg Tech 1997;5:277-38.

4. Schur MO, Breitwieser H, Melzer A, Kurnert W, Schmitt M, Voges U, Buess G. Experimental telemanipulation in endoscopic surgery. Surg Laparosc Endosc 1996;3:167-75.

5. Shennib H. Evolving strategies in minimally invasive coronary artery surgery. Int J Cardiol 1997;62:S81-8. 